Emission spectrum of an electric-ionization high-pressure carbon dioxide laser

This article has been downloaded from IOPscience. Please scroll down to see the full text article.

1974 Sov. J. Quantum Electron. 4185

(http://iopscience.iop.org/0049-1748/4/2/A10)

View the table of contents for this issue, or go to the journal homepage for more

Download details:

IP Address: 134.58.253.57

The article was downloaded on 06/05/2013 at 15:37

Please note that terms and conditions apply. 


\title{
Emission spectrum of an electric-ionization high-pressure carbon dioxide laser
}

\section{A. Ageikin, V. N. Bagratashvili, I. N. Knyazev, Yu. A. Kudryavtsev, and V. S. Letokhov}

(Submitted August 10, 1973)

Kvant. Elektron. (Mosc.), 1, 334-340 (February 1974)

\begin{abstract}
An investigation was made of the emission spectrum of an electric ionization high-pressure $\mathrm{CO}_{2}$ laser. The four strongest vibration-rotational transitions were active in a resonator without a dispersive element. The intensities of the lines fluctuated strongly though the total output was relatively stable. Interferometric measurements of the spectral width of the $P(20)$ lines of the $00^{\circ}-10^{\circ} 0$ band were carried out in a plane-parallel dispersive resonator with a diffraction grating. The width of this line increased with the gas pressure and with the excess of the pumping level over the threshold.
\end{abstract}

\section{INTRODUCTION}

Considerable progress has been made in recent years in the physics of high-pressure molecular gas lasers. The electric ionization excitation method, developed by Basov et al., 1 made it possible to increase the pressure of the active medium in the $\mathrm{CO}_{2}$ laser up to tens of atmospheres. ${ }^{2}$ Recently, an electric discharge $\mathrm{CO}_{2}$ laser ${ }^{3}$ and a highpressure electrochemical HF laser ${ }^{4}$ were developed. This opened up opportunities for building infrared lasers with a tunable frequency using molecular gases at high pressures. This idea was first put forward in ref. 5 (similar ideas were expressed in a more general form in ref. 6).

Continuous frequency tuning of a high-pressure $\mathrm{CO}_{2}$ laser was first reported in ref. 7; the conditions under which such continuous tuning was possible were determined. It was found that the emission spectrum of an electricionization high-pressure $\mathrm{CO}_{2}$ laser consists of many axial modes unless special measures are taken. This presents difficulties in the applications of high-pressure $\mathrm{CO}_{2}$ lasers in the excitation of molecules with narrow absorption lines and in high-resolution infrared spectroscopy. Therefore, it would be very interesting to study the spectral composition of the emission of high-pressure molecular gas lasers.

An experimental study of the emission spectrum of an electric-ionization high-pressure $\mathrm{CO}_{2}$ laser is reported below. We shall show that an increase in the gas pressure, which is necessary for the widening of the range of smooth frequency tuning, also broadens the emission spectrum. The number of the emitted axial modes depends on several experimental parameters, and particularly on the type of

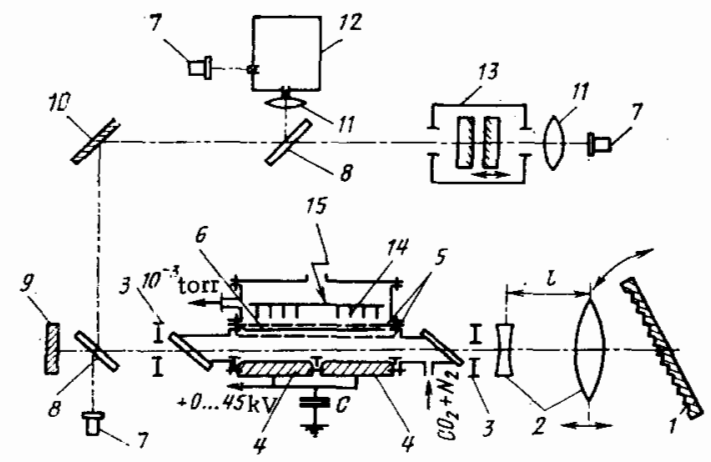

Fig. 1. Schematic diagram of the apparatus: 1) diffraction grating; 2) telescope lenses; 3) screens; 4) metal electrodes; 5) brass grids; 6) polyethylene terephthalate film: 7) thermopiles; 8) $\mathrm{NaCl}$ plates; 9) plane metal mirror; 10) mirror; 11) focusing lenses; 12) IKM-1 monochromator; 13) Fabry-Perot interferometer; 14) field-emission cathodes; 15) high-voltage pulse produced by a coaxial Blumlein line. resonator, excess of the pumping level over the threshold, and pressure of the working mixture. Such features of the laser action as the multimode emission, competition between various laser transitions, etc., suggest that highpressure $\mathrm{CO}_{2}$ lasers should have spectral characteristics similar to those of solid-state lasers. We shall conclude by describing measures for ensuring the minimum width of the emission spectrum, which consists of just one or two axial modes.

\section{A.PPARATUS}

The apparatus is shown schematically in Fig. 1. The preliminary ionization of the gas in the laser enclosure was performed by a fast-electron beam $(\sim 200-250 \mathrm{keV})$ in the form of pulses of $15 \mathrm{nsec}$ duration and 100-200 A amplitude. The evacuated acceleration chamber was separated from the high-pressure laser cell by a polyethylene terephthalate film $85 \mu$ thick. A non-self-sustaining discharge in the working chamber was excited by a $0.1 \mu \mathrm{F}$ capacitor with a $\sim 30 \mathrm{nH}$ intrinsic inductance, charged to a voltage of $0-45 \mathrm{kV}$. The discharge was excited between a brass grid and a sectioned plane polished electrode with rounded edges. The distance between these electrodes was $9 \mathrm{~mm}$ and the active length was $30 \mathrm{~cm}$. The capacitor was partly discharged, producing current pulses of $\sim 2 \mathrm{kA}$ amplitude and $0.5 \mu \mathrm{sec}$ duration. The pumping energy was deduced from the difference between the initial and final voltages across the capacitor. The laser was excited at a repetition frequency of $1-0.05 \mathrm{~Hz}$. The gas pressure in the working cell was varied in the range up to 6 atm. Usually a mixture of the $\mathrm{N}_{2}: \mathrm{CO}_{2}=2: 1$ composition was employed.

Polished stainless-steel mirrors and echelettes with 100 lines $/ \mathrm{mm}$, a blaze angle of $30^{\circ}$, and a first-order reflection coefficient of $90 \%$ were used in the resonator. The grating was used in the autocollimation configuration and it was rotated smoothly about a vertical axis, setting the angle to within $2^{\prime \prime}$. The angular dispersion of the resonator with the echelette was increased using a Galilean telescope with a magnification of 3.4 , a relative aperture $1: 10$, and a variable distance between $\mathrm{NaCl}$ lenses. $\mathrm{A}$ plane metal mirror was also used instead of the echelette. The off-axial modes, including those formed as a result of glancing reflection from the electrodes, were eliminated by screens and by special projections between the sections of the electrode. The distance between the mirror 9 and the axis of rotation of the grating was $90 \mathrm{~cm}$ and the distance between the lenses was $20 \mathrm{~cm}$.

Radiation was extracted by an $\mathrm{NaCl}$ plate, oriented at an angle of about $45^{\circ}$ with respect to the optic axis of 
the laser, and it was then directed to a thermopile (us ed to measure the energy of the laser pulses), an IKM-1 monochromator with another thermopile at the exit, and to a Fabry-Perot interferometer with a mechanical scanning device and a thermopile for measuring the signal. The spectral composition of the radiation was determined and separate laser lines were selected employing a modified IKM-1 monochromator with a prism and an echelette with 100 lines $/ \mathrm{mm}$, which replaced a Littrow mirror. The monochromator was calibrated using the absorption spectrum of $\mathrm{NH}_{3}$ and was fitted with a temperature control system. The spectral width of the individual laser lines was investigated using a Fabry-Perot interferometer with a variable dispersion range (in this case from 0.05 to $1 \mathrm{~cm}^{-1}$ ), a maximum transmission of $30 \%$, and a ratio of the transmission peak width (at half-amplitude) to the dispersion range amounting to 0.15 . The $\mathrm{NaCl}$ interferometer plates were surface-finished to within $\lambda / 5-\lambda / 10$ ( $\lambda$ is the wavelength in the visible range) and coated by a film of tellurium.

\section{EMISSION SPECTRUM OF A SYSTEM \\ WITHOUT A DISPERSIVE ELEMENT}

The emission of a $\mathrm{CO}_{2}$ laser with a plane-parallel resonator without a diffraction grating, observed in the range of working-mixture pressures $p>0.5 \mathrm{~atm}$, consisted of four vibration-rotational lines of the $P$ branch of the $00^{0} 1-10^{0} 0$ transition. At low pressures the strongest line was $P(22)$ (Fig. 2). At pressures $p>2$ atm the ratio of the average intensity $\left\langle I_{J}\right\rangle$ of a line $P(J)$ to the average total emission intensity $\left\langle I_{\text {tot }}\right\rangle=\Sigma\left\langle I_{J}\right\rangle$ was highest for the $P(20)$ line. The $P(16)$ and $P(18)$ lines remained relatively weak. The averaging was carried out using the results of 20 measurements.

A characteristic feature of the emission spectrum was the occurrence of fluctuations of the intensities of the individual laser lines in spite of the relative stability of the total emission intensity. The smallest variation was exhibited by the line with the maximum average intensity. Figure 3 shows the pressure dependences of the relative rms deviation of the intensity $\sigma=\left[\left\langle\left(\mathrm{I}_{\mathrm{J}}-\left\langle\mathrm{I}_{J}\right\rangle\right)^{2}\right\rangle\right]^{1 / 2} /\left\langle\mathrm{I}_{\mathrm{J}}\right\rangle$ of the strongest lines $P(22)$ (curve 1) and $P(20)$ (curve 2), and of the magnitude of the fluctuations $\sigma=\left[\left\langle(\mathrm{I}-\langle\mathrm{I}\rangle)^{2}\right\rangle\right]^{1 / 2} /\langle\mathrm{I}\rangle$ of the total intensity $I=\Sigma I_{J}$ (curve 3). We can see that the total intensity fluctuations are at least five times weaker than the fluctuations of the strongest lines. The weakest lines exhibit even stronger fluctuations.

\section{EMISSION SPECTRUM OF A SYSTEM WITH A DISPERSIVE RESONATOR}

The use of a diffraction grating made it possible to select a particular laser gain profile and to study its spectral width

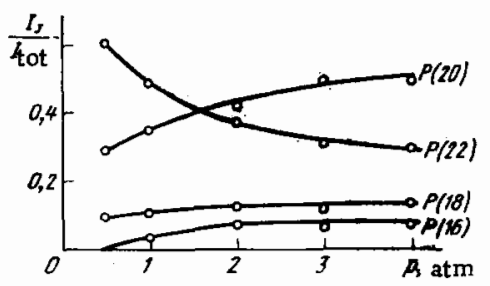

Fig. 2. The dependence of the average relative energy $I_{J} / I_{\text {tot }}$ of a $P(J)$ laser line on the pressure in the $\mathrm{N}_{2}: \mathrm{CO}_{2}=1: 2$ mixture.

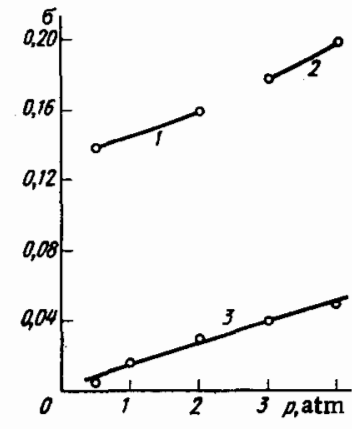

Fig. 3. Pressure dependences of the relative $\mathrm{Ims}$ deviation $\sigma$ of the $P(22)$ line at $\mathrm{P} \leq 2 \mathrm{~atm}(1)$, of the $\mathrm{P}(20)$ line at $p \geq 3$ atm (2), and of the total intensity (3) of the emission from the $\mathrm{CO}_{2}: \mathrm{N}_{2}=1: 2$ mixture.

with a Fabry - Perot interferometer. Figure 4 shows the dependences of the spectral width (measured at half-amplitude) of the $P(20)$ line of the $00^{0} 1-10^{0} 0$ transition on the excess of the excitation energy over the threshold, obtained for different pressures in the working mixture employing a system with a plane-parallel resonator. At $p=1 \mathrm{~atm}$ the width of this line corresponded to two mode spacings and was practically independent of the pumping energy. The mode structure of the spectrum did not appear because the width of the transfer function of the interferometer was $0.008 \mathrm{~cm}^{-1}$, i.e., it was 1.5 times greater than the separation between two neighboring axial modes. When the pressure was increased, the width of the laser lines became greater and began to depend on the excitation energy. Fluctuations of the intensity at the interferometer output were observed, in spite of the stability of the total intensity of the laser radiation, when lines were selected by making the transfer function of the interferometer sufficiently narrow $\left(0.01 \mathrm{~cm}^{-1}\right)$. These fluctuations increased with the pressure in the working mixture. The general behavior of the intensity fluctuations within each emission line was fully analogous to the fluctuations of the intensity of the individual vibration-rotational transitions.

In a resonator with a spherical mirror of $2.5 \mathrm{~m}$ radius of curvature the laser linewidth at $p=1 \mathrm{~atm}$ (Fig. 4) was somewhat greater than at the same pressure in a planemirror resonator. This was also observed at high pressures.

In a plane-mirror dispersive resonator with a telescope in which lenses were separated by the optimal distance the width of the line profile recorded at the exit from the Fabry-Perot interferometer did not increase beyond the width of the transfer function.

\section{DISCUSSION OF RESULTS}

Multimode emission from a laser can be due to various reasons. Under pulsed conditions, multimoding may be due to independent laser action in many axial modes. In this case the width of the spectrum of all the modes is governed by the natural mode selection tending to pull them toward the center of the gain profile..$^{8}$ Under steadystate conditions multimoding may appear because of an inhomogeneous depopulation of the active levels across the spectrum or in space. At high gas pressures the spectral inhomogeneity is absent, but because the rate of diffusion of molecules decreases, a spatial inhomogeneity of the deexcitation may appear in a standing light wave.

The electric ionization laser considered in the present paper emits pulses under conditions resembling fast $Q$

Ageikin et al. 


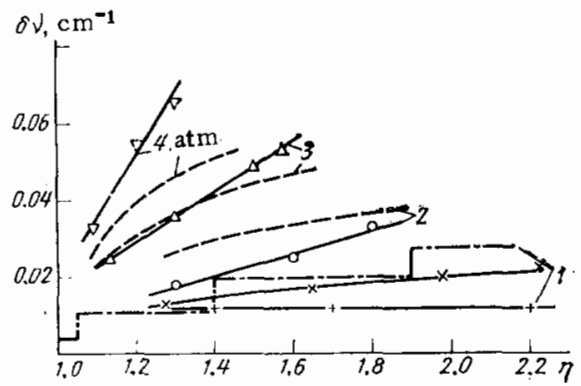

Fig. 4. Dependences of the spectral width $\delta \nu$ of the $P(20)$ laser line of the $00^{\circ} 1-10^{\circ} 0$ transition on the excess of the pumping over the threshold value $\eta=W / W_{\text {th }}$ at different pressures in the working mixture: $\nabla, \Delta, 0,+$ ) experimental results obtained using a plane-mirror resonator; $x$ ) experimental results using a spherical-mirror resonator; the dashed curves represent the results calculated for pulse conditions and the chain curves the results of calculations for steady-state conditions.

switching. The laser action develops at the moment when a sufficiently strong population inversion is reached. After a time the mode intensities rise exponentially and this is followed by the deexcitation of the active medium which is not greatly influenced by the pumping. This model is very appproximate for an electric ionization $\mathrm{CO}_{2}$ laser. It is sufficient to mention that the presence of vibrationally excited $\mathrm{N}_{2}$ molecules can ensure a significant rate of excitation of $\mathrm{CO}_{2}$ for quite a long time. However, the selected transient-process model has hardly any influence on the mode selection. Therefore, in rough estimates this model is quite satisfactory.

The change in the mode intensity distribution $I\left(\nu_{n}, t\right)$ during the linear stage when the gain can be assumed to be constant is described by

$$
I\left(v_{n}, t\right)=I_{0}\left(v_{n}\right) \exp \left[\alpha_{0}\left(v_{n}\right)-\chi\left(v_{n}\right)\right] c t, 0 \leqslant t \leqslant \tau_{d},
$$

where $I_{0}\left(\nu_{n}\right)$ is the initial intensity of a mode $\nu_{n} ; \alpha_{0}\left(\nu_{n}\right)$ is the gain coefficient distributed over the resonator length and corresponding to the frequency $\nu_{\mathrm{n}}\left(\mathrm{cm}^{-1}\right) ; \varkappa\left(\nu_{\mathrm{n}}\right)$ is the frequency-selective minimum loss factor distributed over the resonator length $\left(\mathrm{cm}^{-1}\right)$; $\mathrm{c}$ is the velocity of light in the resonator.

The gain line $\alpha_{0}(\nu)$ and the frequency-selective loss function $x(\nu)$ can be regarded as smooth bell-shaped curves with an effective width at half-amplitude given by

$$
\begin{aligned}
& \Delta v_{g}=\left(\left|\frac{a}{\alpha_{0}} \frac{d^{2} \alpha_{0}(v)}{d y^{2}}\right|\right)^{-1 / 2}, \\
& \Delta v_{l}=\left(\left|\frac{a}{x} \frac{d^{2} x(v)}{d y^{2}}\right|\right)^{-1 / 2},
\end{aligned}
$$

where the derivatives are taken at an extremum. The coefficient $a$ depends on the exact form of the functions $\alpha_{0}(\nu)$ and $x(\nu)$. For example, if the profile is Lorentzian, we have $a=-1 / 8$. Differentiating Eq. (1) twice with respect to the frequency, we obtain the following relationship for the width of the mode spectrum at the end of the linear stage of the laser action:

$$
\Delta v=\left(\frac{1+c \tau_{d} x_{0}}{\Delta v_{g}^{2}}+\frac{c \tau_{d} x}{\Delta v^{2} l}\right)^{-1 / 2}
$$

The duration of the linear stage $\tau_{d}$ can be found from an additional condition

$$
\tau_{d}=\left[c\left(a_{0}-x-\gamma\right)\right]^{-1} \ln \left(I_{\text {sat }} / I_{0}\right),
$$

where $\gamma$ is the nonresonance loss factor per unit length; $I_{\text {sat }}$ is the intensity resulting in the saturation of the gain of the active medium. Equation (3) can be reduced to the form

$$
\Delta v \approx \Delta v_{g}\left\{\frac{(\eta-1) \ln \left(/ \mathrm{sat} I_{0}\right)}{r_{1}+\frac{x}{x+\gamma}\left(\frac{\Delta v g}{\Delta v}\right)^{2}}\right\}^{1 / 2} .
$$

If the population of the upper active level is $10^{17} \mathrm{~cm}^{-3}$ (this corresponds to a gain $\alpha_{0}=2 \cdot 10^{-2} \mathrm{~cm}^{-1}$ ) and the pressure is $\mathrm{p}=3 \mathrm{~atm}$, we find that $\mathrm{I}_{0}=10^{-11} \mathrm{~W}$ per mode. If $\mathrm{I} \approx 10^{4} \mathrm{~W}$ per mode, we find that $\ln \left(\mathrm{I}_{\mathrm{Sat}} / \mathrm{I}_{0}\right)=35$. The effective width of the selective loss function of a dispersive resonator, found experimentally from the dependence of the losses in an equivalent plane-mirror resonator on the angle of rotation of the mirror and from the known angular dispersion of the grating, is $\Delta \nu_{\mathrm{n}} \approx 1 \mathrm{~cm}^{-1}$. A satisfactory agreement with the experimental results is obtained for $p=3 \mathrm{~atm}$. At $\mathrm{p}=4 \mathrm{~atm}$ the calculated values are 1.4 times smaller than the measured values.

The emission of the two strongest rotation-vibrational lines can be explained similarly by incomplete selection of these lines. The effective spectral width of the emission band of the $\mathrm{CO}_{2}$ laser decreases by a factor of 15 , becoming $3.5 \mathrm{~cm}^{-1}$, which corresponds to the emission involving two or three gain lines.

We shall estimate the influence of the spatial inhomogeneity on the excitation of many axial modes in a highpressure gas laser. Such estimates can be made using the theory developed for solid-state lasers if allowance is made for the diffusion of active particles. ${ }^{9}$ The parameter describing the influence of diffusion is

$$
\varepsilon=\left[1+\theta \tau(4 \pi / \lambda)^{2}\right]^{-1},
$$

where $\theta \approx{ }^{1 / 3} \mathrm{v}\left(\mathrm{N}_{0} \Sigma_{\mathrm{el}}\right)=0.8 \mathrm{~cm}^{2} \cdot \mathrm{sec}^{-1} \cdot$ atm is the diffusion coefficient of the active particles; $v$ and $\Sigma_{\text {el }}$ are the velocity and elastic collision cross section of the gas particles; in this case $\tau$ is equal to the vibration - rotational relaxation time of the upper active level: $\tau=\tau_{\mathrm{VT}}=10^{-5}$ $\mathrm{sec} \cdot \mathrm{atm}$.

The diffusion of particles in a high-pressure $\mathrm{CO}_{2}$ laser can be ignored completely at pressures $p>50 \mathrm{~atm}$. Estimates of the width of the laser line, carried out as in ref. 9 for $p=3 \mathrm{~atm}$, are represented by a stepped curve in Fig. 4. The discrepancy between this curve and experimental results is approximately a factor of $2-3$.

The width of the $\mathrm{CO}_{2}$ laser line rises rapidly on increase of the gas pressure and on the increase of the excess of the pumping energy over the threshold value. In order to reduce the width of the laser line, we must use dispersive resonators which have a sufficiently narrow selective loss function. A combination of a telescope and a dispersive resonator with a diffraction grating seems to be particularly promising. 
${ }^{1}$ N. G. Basov, É. M. Belenov, V. A. Danilychev, O. M. Kerimov, I. V. Kovsh, and A. F. Suchkov, ZhETF Pis. Red., 14, 421 (1971) [JETP Lett., 14, 285 (1971)].

${ }^{2}$ N. G. Basov, É. M. Belenov, V. A. Danilychev, O. M. Kerimov, I. B. Kovsh, A. S. Podsosonnyi, and A. F. Suchkov, Zh. Eksp. Teor. Fiz,, 64, 108 (1973) [Sov. Phys. -JETP, 37, 58 (1973)].

'D. J. Hidson, V. Makios, and R. W. Morrison, Phys, Lett. A, 40, 413 (1972).

${ }^{4}$ V. N. Bagratashvill, I. N. Knyazev, Yu. A. Kudryavtsev, and B. S. Letokhov,
ZhETF Pis. Red., 18, 110 (1973) [JETP Lett., 18, 62 (1973)].

${ }^{5}$ V. N. Bagratashvili, I. N. Knyazev, and V. S. Letokhov, Opt. Commun., 4,154 (1971).

${ }^{6}$ N. G. Basov, É. M. Belenov, V. A. Danilychev, and A. F. Suchkov, ZhETF Pis. Red, 14, 545 (1971) [JETP Lett., 14, 375 (1971)].

${ }^{7}$ V. N. Bagratashvili, I. N. Knyazev, Yu. A. Kudryavtsev, and V. S. Letokhov, Opt. Commun., 9, 135 (1973).

${ }^{8}$ W. R. Sooy, A ppl. Phys. Lett.. 7, 36 (1965).

${ }^{9}$ H. Statz, C. L. Tang, and J. M. Lavine, J. Appl. Phys., 35, 2581 (1964). 